\title{
Measure for measure
}

\author{
The redefinition of SI units removes materiality from science's weights and measures. There's logic to the decision, \\ but it reminds us what we still don't know about nature's scales.
}

\section{T} he thirteenth-century Assize of Bread and Ale is not, by today's standards, the most precise prescription for defining weights and measures: "By the Consent of the whole Realm of England, the measure of our Lord the King was made; that is to say, That the English Penny, called a Sterling, round and without clipping, shall weigh 32 Wheat Corns in the midst of the Ear, and $20 \mathrm{~d}$. do make an Ounce, and 12 Ounces one Pound."

However, it testifies to the universal need to agree on what such standards are. Having a consensual system for units of quantification is the only way to do trade without argument. Currency exchange schemes began long ago through comparison of relative amounts of gold and silver in coinage systems, and devaluation of currency occurred because of the aforementioned habit of 'clipping' coins to remove a small amount of the precious metal, compromising the face value.

There is in these early schemes for standardizing measures a reassuring materiality: value comes from the quantity of metal, and that in turn is assayed by comparison with nature's own metrology, as expressed (with what looks now like perilous contingency) in grains of corn. These origins are now themselves ingrained in our cultures and languages: the pound sterling may be a currency unit held on paper or in computer bits, but it's no coincidence that the term refers also to a unit of mass.

Such solidity is about to evaporate, however. This May, the SI unit of mass the kilogram - will no longer be defined by a material object, the International Prototype of the Kilogram (IPK) made of a machined alloy of platinum and iridium and held in a controlled environment by the International Bureau of Weights and Measures in Sèvres, France. Instead it will be defined via a fundamental constant of nature: Planck's constant $h$, which specifies the scale of quantum granularity. The units of $h$ are $\mathrm{kg} \mathrm{m}^{2} \mathrm{~s}^{-1}$ : mass becomes linked to the speed of light and $h$ thanks to Planck's and Einstein's formulae for energy, equal to $h$ times frequency and also to $m c^{2}$.

By redefining mass in this way - a decision taken last November at the General Conference on Weights and Measures after much debate - it becomes immutable, free from the fluctuations, drift and discrepancies to which the IPK and its official reference

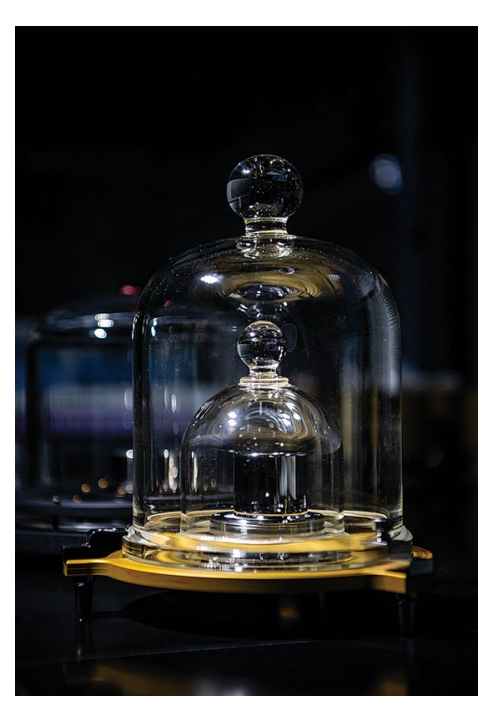

One of the several standard kilograms housed around the world. Credit: The Washington Post/Getty

copies in other countries are prone thanks to tiny chemical changes of the material.

At the same time, three other of the seven SI units will also refer to fundamental constants. The kelvin of the temperature scale is to be defined via Boltzmann's constant $k$; the ampere is tied to the unit of electrical charge $e$; and the mole takes its value from Avogadro's number. These changes also expunge the system of materiality and the need for experimental determination: the kelvin, for instance, was previously related to the triple point of water, and the mole to a given mass of carbon-12. In effect the redefinitions reverse the relationship between a measure and the associated fundamental constant: it's not that the constants, as determined empirically, have uncertainties expressed in particular units, but that that units themselves inherit those empirical limitations.

These SI definitions are interrelated. To get mass from $h$, for example, you need to specify measures of distance and time. The latter is defined by the ground-state hyperfine transition frequency of the caesium-133 atom, and the metre comes from combining this measure of a second with the speed of light in a vacuum (the constant to which the metre was linked in 1983). Boltzmann's constant is yoked to these three definitions of time, distance and mass.

You might argue, then, that material contingency is still embedded in the new SI system: to know these measures, someone somewhere has to get hold of caesium, just as in the thirteenth century they needed wheat and barley. But the gains in precision and stability are obvious: a caesium-133 atom is the same in Paris or Tokyo. The mystery, some might say, is only why this switch took so long, and one can't help but wonder if some resistance to change stemmed from the same kind of unease that accompanied the abandonment of the gold standard during the Great Depression, whereby money seemed no longer anchored by anything tangible.

But one of the deeper consequences of the redefinition is that we are reminded of how nature itself seems to have a bureau of weights and measures, into which we have so far been denied entrance. The 'fundamental constants' bear that label almost as an admission of defeat: currently we have to accept them as a given, taking nature as we find it. Planck's constant sets the scale of any meaningful definition of length, for example: below the distance $\sqrt{h G / 2 \pi c^{3}}=1.6 \times 10^{-35}$ $\mathrm{m}$ (the Planck length, where $G$ is the gravitational constant) we can formulate no coherent notion of space. But why is $h$ so small, even compared to the proton? Why, for that matter, is it not zero, smoothing quantum granularity into classical continuity? Answering those questions would solve some of the most profound puzzles in physics.

But perhaps there is no real answer. Maybe some speculative cosmological theories are correct to say that our fundamental constants are anthropically selected from an arbitrary range within a multiverse: only in universes with these values can there be grains of corn and cylinders of platinum alloy. For others that is a dispiriting idea, confronting the scientific quest for answers with sheer happenstance.

All this is a long way from the business of polishing blocks of metal with alcohol in an effort to give our balances a stable point of reference. Redefining the kilogram doesn't simply mean overturning a convention that has existed since 1889; for the first time it cuts the scales of the greengrocer and the jeweller free from stuff we can touch and see, and forces us to recognize how the reassuring heft of the material world is a puzzle still to be solved.

Published online: 20 March 2019 https://doi.org/10.1038/s41563-019-0333-4 